\title{
Tipificação de Tumores Cerebrais Utilizando Características Radiômicas e Redes Neurais Profundas
}

\author{
Davidson L Souza ${ }^{1}$, Alessandra M Coelho ${ }^{1}$, Matheus F O Baffa ${ }^{2}$ \\ ${ }^{1}$ Departamento Acadêmico da Ciência da Computação \\ Instituto Federal de Educação, Ciência e Tecnologia do Sudeste de Minas Gerais \\ campus Rio Pomba (IF SudesteMG) \\ Rio Pomba - MG - Brasil \\ ${ }^{2}$ Departamento de Computação e Matemática \\ Universidade de São Paulo (USP) \\ Ribeirão Preto - SP - Brasil \\ davidson.lucas.souza@outlook.com
}

\begin{abstract}
The Central Nervous System's tumors consist in the development of cancer cells in one of the nervous structures, such as the brain and the spinal cord. Although there are no routine tests for early diagnosis, the early detection on MRI images can provide a better prognosis. Thus, this paper addresses the development of a brain tumor classification method for multimodality MRI. Using Deep Neural Networks and radiomic feature extraction, the present study obtained a 90,29\% accuracy in determining the types of tumor present in the exams.
\end{abstract}

Resumo. Os tumores do Sistema Nervoso Central consistem no desenvolvimento de células cancerígenas em alguma das estruturas nervosas, como o cérebro e a medula espinhal. Embora não haja exames rotineiros de detecção precoce, a detecção do tumor nas fases iniciais da doença em imagens de Ressonância Magnética pode proporcionar uma melhor expectativa de cura ao paciente. Dessa forma, o objetivo deste trabalho é o desenvolvimento de um método computacional para classificação do tumor cerebral em múltiplas modalidades de Ressonância Magnética. Utilizando Redes Neurais Profundas e extração de características radiômicas, o presente trabalho obteve uma taxa de acerto de 90,29\% em determinar os tipos de tumor presentes nos exames.

\section{Introdução}

O cérebro é o principal órgão que compõe o Sistema Nervoso Central (SNC), sendo responsável por funções como cognição, memória e coordenação motora do nosso corpo. Tumores no cérebro correspondem a um grupo de malignidades causadas pelo crescimento desordenado de células no interior do tecido cerebral e são categorizados de acordo com o tecido de origem [Louis et al. 2016].

Estima-se uma incidência de 2,6 novos casos para cada 100.000 mulheres e 3,6 entre os homens [Bondy et al. 2008]. Tais tumores, por serem assintomáticos em fase inicial, são de difícil diagnóstico e, consequentemente, possuem baixa taxa de sobrevida. Cerca de $88 \%$ dos casos de tumor no cérebro são malignos. No 
Brasil, a taxa de mortalidade é de aproximadamente $87 \%$ dos casos detectados [Instituto Nacional do Cancer. 2021].

A principal abordagem clínica para o diagnóstico consiste em uma análise manual de imagens de ressonância magnética, por profissionais da área. Todavia, tal processo sofre de subjetividade, como cansaço ou inexperiência do profissional em questão. Para reduzir a subjetividade e padronizar o diagnóstico, sistemas de visão computacional vêm sendo propostos a fim de substituir a análise manual [Tandel et al. 2019].

Desta forma, o objetivo deste trabalho é implementar um método de classificação usando aprendizado profundo para o diagnóstico de tumores no cérebro em imagens de ressonância magnética coletadas nas modalidades T1, T2 e FLAIR. Para isso, foram extraídas múltiplas características espaciais baseadas em cor, textura e forma, a fim de descrever o conteúdo semântico da imagem. A principal contribuição deste trabalho é o desenvolvimento do modelo de classificação multiclasse entre exames por imagens de pacientes saudáveis e três diferentes tipos de tumor cerebral (glioma, meningioma e tumor na pituitária) utilizando características radiômicas.

\section{Materiais e Métodos}

\subsection{Base de dados}

A base de dados utilizada possui um dataset de 1184 imagens, divididas nas classes saudável, glioma, meningioma e tumor na pituitária. Tais imagens foram obtidas da base [Bhuvaji et al. 2020], nas modalidades T1, T2 e FLAIR. Optou-se por utilizar apenas imagens de corte axial, para reduzir complexidade em fases iniciais.

\subsection{Extração de Características}

Para extração de características utilizou-se a abordagem radiômica, a qual representa o conteúdo da imagem utilizando múltiplas características qualitativas [Lambin et al. 2012]. A abordagem radiômica reduz a sensibilidade do sistema a ruídos e interferências do paciente ou do equipamento usado para criar as imagens, criando métodos mais robustos e padronizados se comparado com a extração manual de características.

As características radiômicas foram extraídas utilizando a framework PyRadiomics [Van Griethuysen et al. 2017]. Ao todo foram extraídas 313 características, sendo elas características de primeira ordem, como energia e entropia, características de forma, características da matriz de co-ocorrência de nível de cinza, características da matriz de zona do tamanho do nível de cinza, características da matriz de comprimento de execução de nível de cinza, características da matriz de dependência do nível de cinza, dentre outras.

\subsection{Metodologia de Classificação}

A detecção de padrões na base de características extraídas das imagens de Ressonância Magnética foi realizada utilizando uma Rede Neural Profunda (Fully-Connected Neural Network). Para fins de replicabilidade, a Rede Neural proposta foi configurada como se segue. Ao todo foram utilizadas oito camadas, sendo uma camada de entrada, seis camadas ocultas e uma camada de saída. A camada de entrada possui 313 neurônios, 
responsáveis por captar as características e propagá-las na camada seguinte da rede. As camadas ocultas foram compostas por 256 neurônios cada, com a utilização da função de ativação Rectifier Linear Unit (ReLU). Cada camada oculta foi acompanhada de uma operação de dropout, cujo objetivo foi desligar aleatoriamente cerca de $20 \%$ dos neurônios da camada oculta adjacente, auxiliando na criação de um modelo de classificação generalizado, de forma a não ocorrer sobreajuste sobre os dados de treinamento. Por fim, a camada de saída foi composta por quatro neurônios, ilustrando a multi-classificação característica do problema deste trabalho. Na camada de saída utilizou-se a função de ativação Softmax e o algoritmo de otimização Adam. A taxa de erro foi baseada na Categorical Crossentropy.

\section{Resultados e Discussões}

Para o desenvolvimento e avaliação deste trabalho, foi utilizado um computador AMD Ryzen 7, com 16GB de memória RAM, uma GPU NVIDIA GTX 1060 e o sistema operacional Windows 10 Pro. Como ambiente de desenvolvimento utilizou-se Python 3.7, a framework Keras na versão 2.1 e a framework Tensorflow 2.3.

O experimento proposto neste trabalho foi baseado no Holdout Cross-Validation. Nesse protocolo de experimentação, a base de dados é dividida em $80 \%$ para treinamento e $20 \%$ para teste, de forma que os vetores descritores utilizados para o treinamento do modelo não sejam utilizados para a validação.

Para avaliar quantitativamente o modelo de classificação criado, quatro métricas foram calculadas a partir das classificações realizadas sob a base de validação, sendo elas a acurácia, a a área abaixo da curva ROC (Area Under the Curve - AUC), a precisão e a revocação. A Tabela 1 contém os resultados obtidos na avaliação qualitativa.

Tabela 1. Resultados obtidos no experimento proposto.

\begin{tabular}{ccccc}
\hline$\#$ & Acurácia & AUC & Precisão & Revocação \\
\hline Resultados & $90,29 \%$ & $95,61 \%$ & $93,53 \%$ & $90,12 \%$ \\
\hline
\end{tabular}

O método proposto apresentou resultados satisfatórios ao problema multiclasse apresentado. De modo geral, a taxa de acerto do algoritmo foi de 90,29\%, mostrando que as características radiômicas aliadas à detecção de padrões das Redes Neurais Profundas podem auxiliar de forma eficaz na detecção de três diferentes tumores do cérebro. A Tabela 2 contém a matriz de confusão, que avalia a performance da classificação em cada classe.

Tabela 2. Matriz de confusão contendo as predições de cada classe.

\begin{tabular}{|c|c|c|c|c|}
\hline$\#$ & Glioma & Meningioma & Saudável & Pituitária \\
\hline Pred. Glioma & 50 & 1 & 4 & 1 \\
\hline Pred. Meningioma & 5 & 69 & 0 & 1 \\
\hline Pred. Saudável & 4 & 2 & 46 & 1 \\
\hline Pred. Pituitária & 6 & 1 & 0 & 46 \\
\hline
\end{tabular}




\section{Conclusão}

O cérebro é o principal órgão do nosso SNC. Apesar da baixa taxa de ocorrência, os tumores cerebrais possuem alta taxa de mortalidade, podendo chegar a até $87 \%$ dos casos detectados. Como inexistem métodos rotineiros para o diagnóstico precoce, o uso de técnicas computacionais para o auxílio ao diagnóstico do câncer no SNC pode auxiliar na detecção de mais casos, a partir de imagens de ressonância da magnética. Neste trabalho foi proposto o desenvolvimento de um algoritmo de classificação, baseado em Redes Neurais Profundas e na extração de características radiômicas. De forma satisfatória, o método proposto detecta três tipos de câncer, além de exames provenientes de pacientes saudáveis em múltiplas modalidades, como T1, T2 e FLAIR. Em trabalhos futuros, pretende-se desenvolver um sistema de auxílio ao diagnóstico do câncer de SNC.

\section{Agradecimentos}

Os autores agradecem ao IF SudesteMG e ao Programa de Educação Tutorial (PET Conexões de Saberes Ciência da Computação) pelo apoio técnico e financeiro para o desenvolvimento do projeto.

\section{Referências}

Bhuvaji, S., Kadam, A., Bhumkar, P., Dedge, S., and Kanchan, S. (2020). Brain tumor classification (mri). Kaggle, doi: 10.34740/kaggle/dsv/1183165.

Bondy, M. L., Scheurer, M. E., Malmer, B., Barnholtz-Sloan, J. S., Davis, F. G., Il'Yasova, D., Kruchko, C., McCarthy, B. J., Rajaraman, P., Schwartzbaum, J. A., et al. (2008). Brain tumor epidemiology: consensus from the brain tumor epidemiology consortium. Cancer, 113(S7): 1953-1968.

Instituto Nacional do Cancer. (2021). Câncer do sistema nervoso central. Disponível em: $\quad<$ https://www.inca.gov.br/tipos-de-cancer/cancer-do-sistema-nervoso-central $>$. Acesso em: 29 mai. 2021.

Lambin, P., Rios-Velazquez, E., Leijenaar, R., Carvalho, S., Van Stiphout, R. G., Granton, P., Zegers, C. M., Gillies, R., Boellard, R., Dekker, A., et al. (2012). Radiomics: extracting more information from medical images using advanced feature analysis. European journal of cancer, 48(4):441-446.

Louis, D. N., Perry, A., Reifenberger, G., Von Deimling, A., Figarella-Branger, D., Cavenee, W. K., Ohgaki, H., Wiestler, O. D., Kleihues, P., and Ellison, D. W. (2016). The 2016 world health organization classification of tumors of the central nervous system: a summary. Acta neuropathologica, 131(6):803-820.

Tandel, G. S., Biswas, M., Kakde, O. G., Tiwari, A., Suri, H. S., Turk, M., Laird, J. R., Asare, C. K., Ankrah, A. A., Khanna, N., et al. (2019). A review on a deep learning perspective in brain cancer classification. Cancers, 11(1):111.

Van Griethuysen, J. J., Fedorov, A., Parmar, C., Hosny, A., Aucoin, N., Narayan, V., Beets-Tan, R. G., Fillion-Robin, J.-C., Pieper, S., and Aerts, H. J. (2017). Computational radiomics system to decode the radiographic phenotype. Cancer research, 77(21):e104-e107. 